\title{
Análise fatorial exploratória e hierárquica da Escala Triangular do Amor ${ }^{1}$
}

\author{
José Augusto Evangelho Hernandez² \\ Universidade do Estado do Rio de Janeiro, Rio de Janeiro-RJ, Brasil
}

\section{RESUMO}

As análises fatoriais da Escala Triangular do Amor de Sternberg (ETAS), que teoricamente possui três componentes, têm extraído um primeiro fator que explica a maior parte da variância, revelado cross loadings em diversos itens e elevadas correlações entre fatores. Alguns pesquisadores postularam a unidimensionalidade da medida. Outros tentando superar os problemas psicométricos definiram versões reduzidas (ETAS-R). O estudo atual reexaminou a ETAS e a ETAS-R com duas amostras de 937 e 817 do sul e do sudeste do Brasil, respectivamente, buscando contribuir para a clarificação da complexidade fatorial das escalas. Os escores foram submetidos às análises fatoriais exploratórias e hierárquicas. Para a ETAS apareceram fortes evidências da existência de uma dimensão geral em detrimento das dimensões específicas e para a ETAS-R, uma solução de segunda ordem e três fatores de primeira ordem com itens representando equilibradamente as dimensões geral e específica.

Palavras-chave: amor; paixão; intimidade; psicometria.

\section{ABSTRACT - Exploratory and hierarchical factorial analysis of the Triangular Love Scale}

The factorial analyses of Sternberg's Triangular Love Scale (ETAS), which theoretically has three factors, have extracted a first factor that explains most of the variance, revealed cross loadings in various items and high correlations between factors. Several researchers have postulated the unidimensionality of the measure. Others, in trying to overcome these psychometric problems, have defined reduced versions (ETAS-R). The current research reviewed the ETAS and ETAS-R (short version) with two samples of convenience - with 937 and 817 participants, from the South and Southeast regions of Brazil, respectively - seeking to contribute to the clarification of this factorial complexity. The scores were submitted to Exploratory and Hierarchical Factor Analyses. For ETAS, strong evidence appeared of the existence of a general factor to the detriment of specific dimensions. For ETAS-R, a second-order and three first-order factors, with items presenting general and specific dimensions in balance.

Keywords: love; passion; intimacy; psychometrics.

\section{RESUMEN - Análisis factorial exploratoria y jerárquica de la Escala Triangular del Amor}

El análisis factorial de la Escala Triangular del Amor de Sternberg (ETAS), que teóricamente tiene tres componentes, há extraído un primer factor que explica la mayor parte de la variabilidad, mostrando cargas cruzadas en varios ítems y altas correlaciones entre los factores. Algunos investigadores solicitaron la unidimensionalidad de la medida. Otros, en el intento de superar los problemas psicométricos definieron versiones reducidas (ETAS-R). El estudio actual reexaminó la ETAS y ETAS-R con dos muestras de 937 y 817 individuos, del sur y sudeste de Brasil respectivamente, buscando contribuir al esclarecimiento de la complejidad factorial de las escalas. Los resultados fueron sometidos al Análisis Factorial Exploratorio y Jerárquico. Para la ETAS surgieron fuertes evidencias de la existencia de una dimensión general en prejuicio de las dimensiones específicas y para la ETAS-R, una solución de segundo orden y tres factores de primer orden con ítems que representan equilibradamente las dimensiones generales y específicas.

Palabras clave: amor; pasión; intimidad; psicometría.

O embrião da Teoria Triangular do Amor está no trabalho de Sternberg e Grajek (1984), que procuraram definir e comparar a natureza do amor de acordo com estruturas teóricas das teorias psicométricas da inteligência de Spearman, Thomson e Thurstone. Os resultados sugeriram que o amor é mais bem caracterizado pelo modelo thomsoniano, ainda que possa ser sentido como com uma coisa só, não é uma coisa só. Quando alguém ama, experimenta um conjunto de sentimentos, desejos e pensamentos que, na totalidade, leva à percepção da unicidade.

Por meio da revisão da literatura norte-americana e de outros países, Sternberg (1986) chegou à conclusão que, embora alguns elementos do amor dependessem parcialmente da época e cultura, a Paixão, a Intimidade e a Decisão/Compromisso, em 
geral, transcenderiam as elas. Assim, propôs a Teoria Triangular do Amor, adotando a figura geométrica do triângulo como metáfora para representar esses três componentes do amor.

A Paixão é o elemento romântico e físico do relacionamento amoroso. Este pode ser descrito como: a sensação romântica, a atração física, o desejo de estar junto, a satisfação e o contentamento sexual mútuo e a excitação física e emocional. A Intimidade existe nos relacionamentos em que está presente o desejo de promover o bem-estar da outra pessoa, a felicidade de poder compartilhar experiências de vida, o senso de respeito mútuo, a capacidade de contar com a pessoa amada em momentos de necessidade, a compreensão mútua da participação nos momentos difíceis da vida do parceiro, a compreensão íntima compartilhada de sintonização, a disposição de dar apoio emocional um ao outro, a recepção de apoio emocional da pessoa amada, a capacidade de se comunicar além dos níveis superficiais ou práticos e o reconhecimento mútuo do valor do parceiro. A Decisão/compromisso é caracterizada por duas fases: a decisão de que amamos alguém e o compromisso de manter esse amor. Esse processo é essencial para um relacionamento duradouro e inclui a certeza de que o sentimento é especial, a disposição para simbolizar e articular esse amor de algum modo, a compreensão de que é mais do que um capricho passageiro e a decisão de investir ao longo do tempo (Sternberg, 1986; 1989; 1997; 2006).

A partir das medidas de amor de Rubin (1970) e Levinger, Rands, e Talaber (1977), Sternberg (1988) conduziu a primeira pesquisa para desenvolver uma medida para a sua teoria, a Escala Triangular do Amor de Sternberg (ETAS) que continha 36 itens, 12 itens para cada uma das subescalas: Intimidade, Paixão e Decisão/Compromisso. Os 84 participantes, homens e mulheres, que tinham de 19 a 62 anos de idade $(M=28$; $D P=8$ ) e estavam envolvidos em relacionamentos amorosos heterossexuais com tempo de duração de um mês a 22 anos $(M=4,5 ; D P=4,6)$, responderam a ETAS pensando em seis tipos de relacionamentos de amor: com a mãe, com o pai, com irmão mais próximo em idade, com amante/esposo, com o melhor amigo do mesmo sexo e com um amante/esposo ideal. Os resultados nem sempre corroboraram a teoria, as correlações entre os três componentes foram maiores do que se esperava e alguns itens não mediram o que supostamente deveriam medir.

Hendrick e Hendrick (1989) testaram uma versão da ETAS de 45 itens fornecida por Sternberg. Nos resultados, o forte primeiro fator foi coerente com as elevadas correlações entre as subescalas e a superposição das cargas fatoriais dos itens entre os três fatores. Nas conclusões, os pesquisadores questionaram se as subescalas efetivamente avaliavam construtos específicos ou se, principalmente, avaliavam um construto único.

Os resultados de Carreño e Serrano (1995) foram equivalentes aos encontrados por Hendrick e Hendrick (1989) e consideraram a possibilidade de se pensar em uma ETAS unifatorial. Além destes, resultados semelhantes foram encontrados por Acker e Davis (1992), Aron e Westbay (1996), Chojnacki e Walsh (1990) e Whitley (1993).

Sternberg apresentou um segundo estudo de validação de construto da ETAS, na tentativa de sanar os problemas encontrados antes (Sternberg, 1988). A ETAS foi revisada, alguns itens foram excluídos e outros novos incluídos. Os 101 participantes responderam a uma ETAS de 45 itens, 15 para cada subescala. As mudanças reduziram o número de itens que saturavam em fatores para os quais não foram designados e as correlações entre as subescalas. Os resultados não foram perfeitos, mas forneceram mais evidências de validade de construto para a ETAS (Sternberg, 1997).

Graham (2011) realizou uma meta-análise com modelagem de equações estruturais em 103 amostras de 88 estudos, envolvendo 19.387 indivíduos e abrangendo 40 anos de investigações sobre o amor. Esse estudo identificou os fatores subjacentes à Escala de Amar e Gostar de Rubin (1970), à Escala de Amor Apaixonado de Hatfield e Sprecher (1986), à Escala de Atitudes de Amor de Hendrick e Hendrick (1986) e à ETAS, consideradas as medidas mais populares de amor. Foram extraídos três fatores, o primeiro foi mais consistente e identificado como Amor e as três subescalas da ETAS saturaram suas cargas fatoriais nele. Graham (2011) concluiu que a Teoria Triangular do Amor fornece um modelo elegante que combina os componentes Paixão, Intimidade e Decisão/Compromisso, mas os dados sugeriram que eles não são distintos uns dos outros.

A primeira adaptação brasileira da ETAS foi publicada por Hernandez (1999). Cassep-Borges e Teodoro (2007) fizeram uma nova tradução da medida e buscaram evidências de validade para ela. Essas pesquisas encontraram evidências de uma estrutura fatorial de três fatores e fortes consistências internas para as escalas, mas os problemas psicométricos relatados nos estudos anteriores persistiram. Cassepp-Borges e Pasquali (2012, p.25) chamaram a atenção para o fato de que "provavelmente existe um fator geral" e reconheceram que a medida apresenta itens complexos que carregam fortes em mais de um fator.

Buscando amenizar os problemas apresentados, foram ajustadas versões reduzidas dela (ETAS-R) por meio da exclusão de itens com desempenho psicométrico fraco (Acker \& Davis, 1992; Lemieux \& Hale, 2000). No Brasil, Cassep-Borges e Teodoro (2007) propuseram uma ETAS-R com 18 itens. Gouveia, Fonseca, Cavalcanti, Diniz, e Dória (2009) também definiram uma ETAS-R, 
selecionando os 15 melhores itens de Hernandez (1999). Nesses estudos, as saturações de itens em subescalas para as quais não foram designados foram superadas, mas as elevadas correlações entre as subescalas persistiram. Posteriormente, foram realizadas análises fatoriais confirmatórias com a ETAS-R de Cassepp-Borges e Teodoro (2007) e os resultados encontrados foram semelhantes (Andrade, Garcia, \& Cassep-Borges, 2013; CasseppBorges \& Pasquali, 2014).

Apesar dos problemas psicométricos apresentados, a teoria Triangular do Amor é reconhecida como um marco teórico importante no estudo das relações amorosas. Até o presente momento, muitos estudos reconheceram a presença de uma dimensão geral e três dimensões específicas para a ETAS, no entanto, estes não têm precisado a responsabilidade de cada uma dessas instâncias de forma independente sobre a variância de cada variável observada. Os dois estudos atuais buscaram contribuir para a clarificação da estrutura fatorial da ETAS e ETAS-R por meio da análise das relações entre os itens que a compõem e seus fatores de primeira e segunda ordem de forma independente.

\section{Estudo 1}

Nesse estudo foi examinada a ETAS por meio de Análise Fatorial Exploratória e Hierárquica com o objetivo de clarificar a sua estrutura fatorial considerada complexa.

\section{Método}

\section{Participantes}

Foi feita uma amostra de conveniência de 937 indivíduos, 510 (54,4\%) mulheres e 427 (45,6\%) homens da cidade de Porto Alegre e região metropolitana. A idade média dos participantes foi de 31,2 anos; desvio padrão de 11,15 anos com variação de 14 a 74 anos; sete $(0,7 \%)$ pessoas não forneceram a idade. Todos os participantes estavam envolvidos em uma relação amorosa heterossexual de diversos tipos: $444(47,4 \%)$ em união estável ou casamento, $56(6,0 \%)$ em noivado, $413(44,1 \%)$ em namoro e 24 (2,5\%) não forneceram essa informação. Os participantes mantinham esses relacionamentos, em média, há 8,5 anos com desvio padrão de 8,9 anos e 58 (6,2\%) não forneceram esse dado. Quatrocentos e cinquenta e oito $(48,9 \%)$ participantes residiam juntos com os parceiros, $457(48,8 \%)$ não residiam juntos e $22(2,3 \%)$ não deram a informação. Dos respondentes, $316(33,7 \%)$ tinham filhos na relação, 598 (63,8\%) não tinham filhos na relação e $23(2,5 \%)$ não responderam ao item. Quanto à escolaridade, $43(4,6 \%)$ tinham o ensino fundamental, 245 (26,1\%) tinham o ensino médio, $571(60,9 \%)$ tinham o ensino superior,
68 (7,3\%) tinham pós-graduação e 10 (1,1\%) não deram essa informação.

\section{Instrumento}

Sternberg (2006, p.197) registrou que "a versão completa mais recente da Escala Triangular do Amor pode ser encontrada em Sternberg (1998)". Para o estudo atual, foi feita uma revisão na tradução de Hernandez (1999) a partir dessa versão de Sternberg (1998) e das traduções brasileiras de Cassepp-Borges e Teodoro (2007) e Gouveia et al., (2009), assim alguns dos 45 itens da ETAS foram reformulados. Para responder ao instrumento, os participantes usaram uma escala Likert de cinco pontos, onde 1 é nada verdadeiro, 3 é moderadamente verdadeiro e 5 é totalmente verdadeiro, os pontos intermediários indicaram níveis intermediários de sentimentos.

\section{Procedimentos}

$\mathrm{O}$ projeto de pesquisa foi submetido a exame e aprovado pelo Comitê de Ética em Pesquisa da universidade a que se encontra vinculada mediante o parecer $\mathrm{n}^{\mathrm{o}}$ $97 / 2011$. Todos os participantes foram informados sobre os objetivos da investigação e convidados a ler e assinar um Termo de Consentimento Livre e Esclarecido. Os dados foram coletados em campi universitários, em residências dos participantes, em igrejas, em áreas públicas de lazer e em encontros científicos.

\section{Análise de Dados}

Os dados foram submetidos à Análise fatorial exploratória (AFE) no programa Factor 9.3.1 (Lorenzo-Seva \& Ferrrando, 2013), com método de extração unweighted least squares (ULS) e rotação direct oblimin. Para auxiliar na retenção de fatores, utilizou-se a Análise Paralela Clássica (Horn, 1965).

Os dados também foram analisados por meio da solução Schmid-Leiman (SSL, Schmid \& Leiman, 1957) com o objetivo de gerar evidências da existência de um fator geral de segunda ordem e clarificar suas relações com os fatores de primeira ordem e as variáveis observadas. A SSL é uma transformação das matrizes de cargas fatoriais obtidas na análise fatorial para gerar uma estrutura hierárquica em que o fator geral é ortogonal aos fatores específicos.

Essa técnica forneceu informações complementares revelando a influência dos fatores de primeira ordem (F1, F2 e F3) e de segunda ordem (G1), de forma independente e não sobreposta, sobre as variáveis observadas (Figura 1), que podem ajudar na interpretação de fatores de diferentes níveis e revelar a estrutura hierárquica das variáveis (Wolff \& Preising, 2005). Também foi calculado o coeficiente alfa de Cronbach para verificar a consistência interna da ETAS e suas subescalas. 


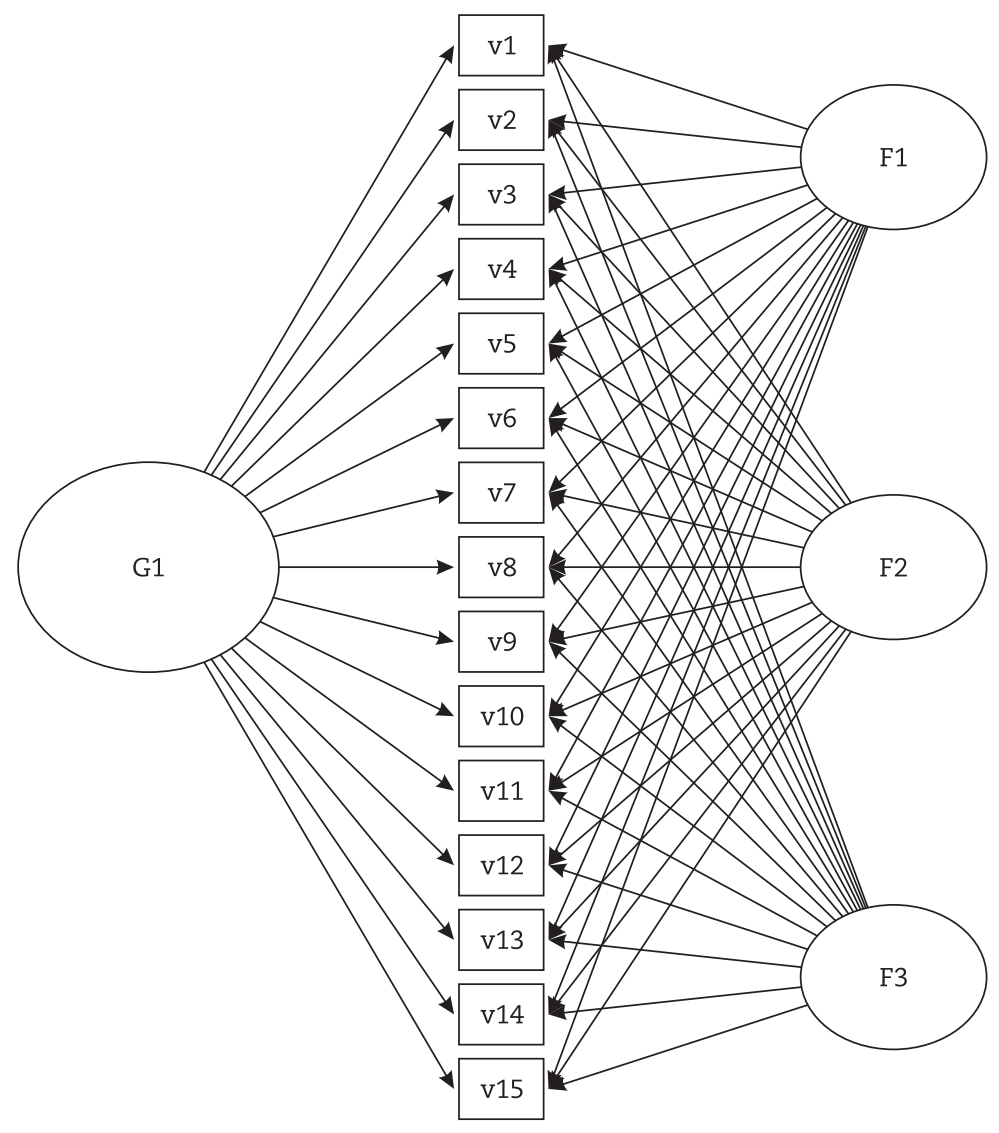

Figura 1. Diagrama ilustrativo do modelo da solução Schmid-Leiman

\section{Resultados}

Do total geral de escores dos participantes na ETAS, $0,4 \%$ estavam ausentes e foram repostos pela média da série. A análise descritiva das variáveis observadas revelou assimetrias negativas moderadas nas distribuições dos escores e não foram encontrados valores extremos. A adequação dos dados à análise fatorial foi verificada por meio do teste Kaiser-Meyer-Olkin que forneceu um valor de 0,98 , considerado excelente. O teste de esfericidade de Bartlett apresentou os valores $\chi^{2}(990)=31.281,85$; $p<0,001$, que permitiram rejeitar a hipótese nula e confirmaram a utilização do método de análise para os dados coletados.

A AFE com método de extração de ULS e rotação direct oblimin revelou uma solução fatorial inicial de cinco fatores com autovalores $>1,0$, que explicaram juntos $62,8 \%$ da variação total, sendo o primeiro fator responsável por $47,5 \%$ dela e os subsequentes $5,5 \%, 4,3 \%$, $2,9 \%$ e $2,6 \%$, respectivamente. No entanto, a análise paralela de Horn (1965) recomendou a retenção de três fatores. Os autovalores empíricos foram 21,34, 2,44, 1,93 e 1,30, e os aleatórios foram 1,44, 1,40, 1,37 e 1,33, nessa ordem. Foram utilizadas 500 matrizes de correlação aleatórias.
Nas análises das matrizes fatoriais neste estudo, foi considerado como critério mínimo de significância para as cargas fatoriais nos itens valores $\geq 0,30$ (Hair, Black, Babin, Anderson, \& Tatham, 2009). No fator 1, carregaram os 15 itens designados para a subescala Decisão/Compromisso $(4,7,8,12,13,18,20,27,28,32,33,35,39,43$ e 44), porém o item 8 carregou também com igual intensidade no fator 3 (Intimidade). No fator 2, carregaram 10 itens da subescala Paixão (3, 5, 9, 11, 23, 29, 31, 37, 41 e 45), mas os cinco itens restantes $(14,16,17,22$ e 34$)$ carregaram mais forte no fator 1 (Decisão/Compromisso). Dos itens designados para a Intimidade, sete carregaram no fator 3 $(6,19,30,36,38,40$ e 42$)$, três carregaram nos fatores $2 \mathrm{e}$ $3(1,2$ e 24$)$ e cinco carregaram no fator $1(10,15,21,25$ e 26). No geral, 14 dos 45 itens da ETAS apresentaram cargas fatoriais com significância prática em mais de um fator ou saturaram mais forte em fator para o qual não foram teoricamente designados (Tabela 1).

Os alfas de Cronbach encontrados para a ETAS total e suas subescalas foram todos excelentes, revelando elevada consistência interna para todas as dimensões do instrumento (Tabela 1). A correlação média interitens foi 0,45 , variando de 0,09 a 0,80 . A correlação item total variou de 0,43 a 0,82 . As correlações entre as três subescalas e a escala total foram fortes e variaram de 0,81 a 0,95 . 
Tabela 1

Matriz de Cargas Fatoriais e Comunalidades da ETAS

\begin{tabular}{|c|c|c|c|c|}
\hline \multirow{2}{*}{ Itens } & \multicolumn{3}{|c|}{ Fator } & \multirow{2}{*}{$h^{2}$} \\
\hline & 1 & 2 & 3 & \\
\hline 15. Estou disposto a compartilhar a mim mesmo e a meus bens com... & 0,87 & $-0,03$ & $-0,09$ & 0,64 \\
\hline 13. Espero amar... por toda minha vida. & 0,85 & 0,07 & $-0,08$ & 0,70 \\
\hline 34. Eu não consigo imaginar a vida sem... & 0,83 & 0,04 & $-0,05$ & 0,67 \\
\hline 33. Eu vejo meu relacionamento com... como permanente (duradouro). & 0,80 & 0,00 & 0,06 & 0,71 \\
\hline 28. Não imagino terminar o relacionamento com... & 0,78 & 0,05 & $-0,03$ & 0,63 \\
\hline 32. Estou seguro (a) do meu amor por... & 0,76 & 0,24 & $-0,09$ & 0,75 \\
\hline 22. Não existe nada mais importante para mim do que minha relação com... & 0,76 & 0,12 & $-0,08$ & 0,62 \\
\hline 27. Eu vejo meu compromisso com... como algo sólido. & 0,74 & $-0,01$ & 0,13 & 0,67 \\
\hline 18. Eu não deixaria nada interferir no meu compromisso com... & 0,72 & 0,14 & $-0,03$ & 0,63 \\
\hline 16. Eu não consigo imaginar que outra pessoa possa me fazer tão feliz como... me faz. & 0,71 & 0,20 & $-0,04$ & 0,66 \\
\hline 20. Eu sempre sentirei uma grande responsabilidade por... & 0,67 & $-0,15$ & 0,12 & 0,46 \\
\hline 44. Mesmo quando é difícil lidar com..., continuo comprometido com o nosso relacionamento. & 0,62 & $-0,05$ & 0,07 & 0,40 \\
\hline 12. Eu não poderia deixar que nada interferisse de algum modo meu compromisso com... & 0,62 & 0,09 & $-0,06$ & 0,40 \\
\hline 43. Eu pretendo continuar meu relacionamento com... & 0,62 & 0,10 & 0,20 & 0,68 \\
\hline 7. Eu estou comprometido em manter a relação com... & 0,58 & $-0,08$ & 0,18 & 0,45 \\
\hline 39. Eu tenho um sentimento de responsabilidade para com... & 0,57 & $-0,10$ & 0,23 & 0,46 \\
\hline 14. Eu idealizo... & 0,56 & 0,27 & 0,07 & 0,63 \\
\hline 25. Eu valorizo muito... em minha vida. & 0,55 & 0,18 & 0,20 & 0,66 \\
\hline 26. Eu me sinto próximo de... & 0,53 & 0,20 & 0,16 & 0,60 \\
\hline 35. Eu considero que minha relação com... foi uma boa decisão. & 0,53 & 0,14 & 0,27 & 0,66 \\
\hline 17. Eu prefiro estar com... do que com qualquer outra pessoa. & 0,52 & 0,38 & 0,00 & 0,63 \\
\hline 4. Eu sei que me importo com... & 0,32 & 0,10 & 0,18 & 0,26 \\
\hline 21. Eu dou considerável apoio emocional a... & 0,31 & 0,04 & 0,27 & 0,30 \\
\hline 10. ...pode contar comigo em momentos de necessidade. & 0,30 & $-0,05$ & 0,24 & 0,21 \\
\hline 3. Só em olhar para... é excitante. & $-0,04$ & 0,67 & $-0,03$ & 0,41 \\
\hline 11. Acho... muito atraente. & 0,12 & 0,66 & $-0,01$ & 0,53 \\
\hline 45. Eu tenho fantasias com... & 0,08 & 0,65 & $-0,06$ & 0,46 \\
\hline 37. Meu relacionamento com... é apaixonante. & 0,19 & 0,61 & 0,18 & 0,72 \\
\hline 23. Eu particularmente gosto do contato físico com... & 0,05 & 0,59 & 0,04 & 0,41 \\
\hline 9. Meu relacionamento com... é muito romântico. & $-0,04$ & 0,56 & 0,36 & 0,57 \\
\hline 5. Com frequência, durante o dia, me descubro pensando em... & 0,08 & 0,54 & 0,07 & 0,39 \\
\hline 41. Quando assisto filmes românticos e leio livros românticos, penso em... & 0,31 & 0,53 & $-0,05$ & 0,52 \\
\hline 31. Eu adoro... & 0,28 & 0,51 & 0,18 & 0,67 \\
\hline 29. Existe algo quase "mágico" no meu relacionamento com... & 0,23 & 0,50 & 0,07 & 0,48 \\
\hline 19. Eu recebo considerável suporte emocional de... & 0,05 & 0,15 & 0,65 & 0,57 \\
\hline 40. Sinto que realmente posso confiar em... & 0,19 & $-0,08$ & 0,64 & 0,54 \\
\hline 6. Posso contar com... na hora que eu necessitar. & 0,11 & $-0,09$ & 0,62 & 0,43 \\
\hline 38. Sinto que... realmente me compreende. & 0,05 & 0,25 & 0,59 & 0,59 \\
\hline 24. Eu me comunico bem com... & $-0,17$ & 0,39 & 0,57 & 0,49 \\
\hline 36. Eu sinto que realmente compreendo... & 0,21 & 0,09 & 0,49 & 0,48 \\
\hline 8. Eu tenho confiança na estabilidade da minha relação com... & 0,40 & $-0,15$ & 0,49 & 0,54 \\
\hline 30. Eu tenho uma relação confortável com... & 0,20 & 0,22 & 0,45 & 0,53 \\
\hline 2. Eu tenho uma relação afetuosa com... & 0,10 & 0,37 & 0,42 & 0,54 \\
\hline 1. Eu promovo ativamente o bem-estar de... & 0,22 & 0,36 & 0,37 & 0,62 \\
\hline 42. Eu compartilho informações pessoais profundas sobre mim mesmo com... & 0,25 & 0,19 & 0,32 & 0,40 \\
\hline Variância comum explicada & 13,3 & 5,9 & 5,3 & \\
\hline Variância comum explicada (\%) & 54,3 & 24,1 & 21,6 & \\
\hline alfas de Cronbach & 0,94 & 0,94 & 0,92 & \\
\hline Alfas ETAS & 0,97 & & & \\
\hline
\end{tabular}

Nota. Método de extração Unweighted Least Squares. Método de rotação Direct Oblimin com normalização de Kaiser. Rotação convergida em 10 iterações. F1=Decisão/Compromisso, F2=Paixão, F3=Intimidade 
$\mathrm{Na}$ sequência, foi realizada uma exploração dos dados com a SSL (Schmid \& Leiman, 1957). Os resultados da SSL, conforme podem ser observados na Tabela 2, revelaram que as cargas fatoriais do fator G1 de segunda ordem são mais elevadas do que as cargas fatoriais dos fatores F1, F2 e F3 de primeira ordem sobre a maioria dos itens da ETAS, excetuando os itens 3, 23 e 45 em que há predomínio das cargas fatoriais do F2 e, no item 6, em que estão igualadas as cargas de $\mathrm{F} 3$ e de $\mathrm{G} 1$.

Tabela 2

Matriz de Cargas Fatoriais da SSL para os Itens e Fatores da ETAS

\begin{tabular}{|c|c|c|c|c|}
\hline Itens & F1 (Decisão/Compromisso) & F2 (Paixão) & F3 (Intimidade) & G1 (Amor) \\
\hline 1 & 0,08 & 0,27 & 0,28 & 0,68 \\
\hline 2 & 0,04 & 0,27 & 0,32 & 0,60 \\
\hline 3 & $-0,00$ & 0,52 & $-0,02$ & 0,37 \\
\hline 4 & 0,12 & 0,04 & 0,12 & 0,48 \\
\hline 5 & 0,04 & 0,40 & 0,05 & 0,46 \\
\hline 6 & 0,03 & $-0,09$ & 0,46 & 0,46 \\
\hline 7 & 0,20 & $-0,09$ & 0,14 & 0,62 \\
\hline 8 & 0,13 & $-0,14$ & 0,38 & 0,61 \\
\hline 9 & $-0,01$ & 0,43 & 0,27 & 0,56 \\
\hline 10 & 0,10 & $-0,07$ & 0,17 & 0,41 \\
\hline 11 & 0,05 & 0,50 & $-0,01$ & 0,52 \\
\hline 12 & 0,21 & 0,04 & $-0,04$ & 0,59 \\
\hline 13 & 0,29 & 0,03 & $-0,05$ & 0,78 \\
\hline 14 & 0,19 & 0,19 & 0,07 & 0,74 \\
\hline 15 & 0,29 & $-0,05$ & $-0,05$ & 0,74 \\
\hline 16 & 0,24 & 0,13 & $-0,02$ & 0,76 \\
\hline 17 & 0,18 & 0,28 & 0,01 & 0,72 \\
\hline 18 & 0,24 & 0,09 & $-0,00$ & 0,75 \\
\hline 19 & 0,01 & 0,10 & 0,48 & 0,56 \\
\hline 20 & 0,22 & $-0,14$ & 0,10 & 0,61 \\
\hline 21 & 0,11 & 0,00 & 0,19 & 0,49 \\
\hline 22 & 0,26 & 0,07 & $-0,05$ & 0,74 \\
\hline 23 & 0,02 & 0,46 & 0,04 & 0,44 \\
\hline 24 & $-0,06$ & 0,29 & 0,43 & 0,46 \\
\hline 25 & 0,19 & 0,12 & 0,16 & 0,76 \\
\hline 26 & 0,18 & 0,12 & 0,12 & 0,73 \\
\hline 27 & 0,25 & $-0,03$ & 0,11 & 0,78 \\
\hline 28 & 0,26 & 0,01 & $-0,02$ & 0,74 \\
\hline 29 & 0,08 & 0,37 & 0,06 & 0,58 \\
\hline 30 & 0,06 & 0,15 & 0,34 & 0,62 \\
\hline 31 & 0,10 & 0,38 & 0,13 & 0,70 \\
\hline 32 & 0,26 & 0,16 & $-0,05$ & 0,81 \\
\hline 33 & 0,27 & $-0,03$ & 0,06 & 0,79 \\
\hline 34 & 0,28 & 0,01 & $-0,03$ & 0,77 \\
\hline 35 & 0,17 & 0,09 & 0,22 & 0,76 \\
\hline 36 & 0,07 & 0,04 & 0,37 & 0,58 \\
\hline 37 & 0,07 & 0,46 & 0,15 & 0,69 \\
\hline 38 & 0,02 & 0,17 & 0,45 & 0,60 \\
\hline 39 & 0,19 & $-0,10$ & 0,18 & 0,62 \\
\hline 40 & 0,05 & $-0,08$ & 0,49 & 0,55 \\
\hline 41 & 0,11 & 0,40 & $-0,03$ & 0,59 \\
\hline 42 & 0,08 & 0,13 & 0,25 & 0,56 \\
\hline 43 & 0,21 & 0,05 & 0,16 & 0,78 \\
\hline
\end{tabular}


Tabela 2 (continuação)

Matriz de Cargas Fatoriais da SSL para os Itens e Fatores da ETAS

\begin{tabular}{ccccc}
\hline Itens & F1 (Decisão/Compromisso) & F2 (Paixão) & F3 (Intimidade) & G1 (Amor) \\
\hline 44 & 0,21 & $-0,06$ & 0,06 & $\mathbf{0 , 6 0}$ \\
45 & 0,04 & $\mathbf{0 , 5 0}$ & $-0,04$ & $\mathbf{0 , 4 5}$ \\
\hline G1 (Amor) & 0,93 & 0,70 & 0,59 & \\
\hline
\end{tabular}

$\mathrm{Na}$ Tabela 2, pode-se observar que as cargas fatoriais de 26 dos 45 itens da ETAS (1,

$4,7,10,12,13,14,15,16,17,18,20,21,22,25,26$, $27,28,32,33,34,35,39,42,43$ e 44) não são suficientes para representar seus construtos ou dimensões específicas (Paixão, Intimidade e Decisão/Compromisso). No entanto, eles apresentam cargas fatoriais que os credenciam como bons representantes do construto geral (Amor).

Por outro lado, as saturações das cargas fatoriais dos 19 itens restantes revelam um razoável equilíbrio entre as dimensões específicas e a dimensão geral. Dessa forma, indicam que representam a dimensão geral, mas também salvaguardam a função de representar suas dimensões específicas, ainda que as cargas fatoriais de apenas três desses superem os valores saturados na dimensão geral. Excetuase desse grupo o item 8 , haja vista que sua carga fatorial não saturou no fator especificado (Decisão/Compromisso) no modelo teórico, contudo é um bom representante do fator geral. Demonstraram evidências empíricas de representarem a Paixão, 10 itens $(3,5$, 9, 11, 23, 29, 31, 37, 41 e 45), e a Intimidade, oito itens (2, 6, 19, 24, 30, 36, 38 e 40). Os 15 itens determinados no modelo teórico para a Decisão/ Compromisso (4, 7, 8, 12, 13, 18, 20, 27, 28, 32, 33, 35, 39,43 e 44) não atingiram a carga fatorial mínima $(0,30)$, revelando-se muito fracos representantes dessa dimensão específica, por outro lado, mostraram-se altamente relacionados com a dimensão geral (Tabela 2).

\section{Estudo 2}

Nesse estudo, foi analisada a ETAS-R versão de Gouveia et al. (2009). O objetivo dessa testagem foi gerar informações complementares sobre a competência dos itens na representação das dimensões subjacentes nessa versão reduzida da medida.

\section{Método}

\section{Participantes}

Foi feita uma segunda amostra de conveniência de 817 indivíduos, 578 (70,7\%) mulheres e 239 (29,3\%) homens, da cidade do Rio de Janeiro e região metropolitana. A idade média dos participantes foi de 31,4 anos, desvio padrão de 11,34 anos. Todos os participantes estavam envolvidos em uma relação amorosa heterossexual, 464 (56,8\%) em união estável ou casamento, 38 $(4,7 \%)$ em noivado, 286 (35\%) em namoro e 29 (3,5\%) não forneceu essa informação. Os participantes mantinham esses relacionamentos, em média, há 7,1 anos, com desvio padrão de 8,1 anos.

\section{Instrumento}

Foi usada ETAS-R produzida por Gouveia et al. (2009) com 15 itens, igualmente divididos entre os componentes Paixão, Intimidade e Decisão/Compromisso. Para responder ao instrumento, os sujeitos usaram uma escala Likert de sete pontos, onde 1 é nada verdadeiro, 4 é moderadamente verdadeiro e 7 é totalmente verdadeiro; os pontos intermediários indicaram níveis intermediários de sentimentos.

\section{Procedimentos}

Foram utilizados os mesmos procedimentos de coleta de dados do Estudo 1.

\section{Análise de Dados}

Os dados foram submetidos às mesmas técnicas $\mathrm{e}$ métodos usados no Estudo 1.

\section{Resultados}

Do total geral de escores dos participantes na ETAS-R, 0,08\% estavam ausentes e foram repostos pela média. A análise descritiva das variáveis observadas revelou assimetrias moderadas nas distribuições dos escores. A adequação dos dados à análise fatorial foi verificada por meio do teste Kaiser-Meyer-Olkin que forneceu um valor de 0,93 , considerado muito bom. O teste de esfericidade de Bartlett apresentou os valores $\chi_{(105)}^{2}=6.470,40 ; p<0,001$, que permitiram rejeitar a hipótese nula e confirmaram a utilização do método de análise para os dados coletados.

A AFE com método de extração de ULS e rotação direct oblimin revelou uma solução fatorial de três fatores com autovalores $>1,0$, que explicaram juntos $64,3 \%$ da variação total, sendo o primeiro fator responsável por 46,7\% dela. A análise paralela clássico de Horn (1965) recomendou a retenção de três fatores. Para os primeiros quatro fatores, os autovalores empíricos foram 6,$995 ; 1,505 ; 1,283$ e 0,715 e os aleatórios foram 1,219; 1,$172 ; 1,135$ e 1,104 , respectivamente. Os percentis 95 dos autovalores aleatórios foram respectivamente: 1,$261 ; 1,203 ; 1,163$ e 1,129. Foram utilizadas 500 matrizes de correlação aleatórias. 
Tabela 3

Matriz de Cargas Fatoriais e Comunalidades da ETAS-R

\begin{tabular}{|c|c|c|c|c|}
\hline Itens & F1 & F2 & F3 & $h^{2}$ \\
\hline 13. Eu não poderia deixar que nada interferisse de algum modo meu compromisso com... & 0,89 & $-0,02$ & $-0,01$ & 0,47 \\
\hline 33. Eu vejo meu relacionamento com... como permanente (duradouro). & 0,83 & 0,10 & $-0,07$ & 0,37 \\
\hline 28. Não imagino terminar o relacionamento com... & 0,75 & 0,06 & $-0,00$ & 0,44 \\
\hline 32. Estou seguro (a) do meu amor por... & 0,74 & $-0,03$ & 0,23 & 0,56 \\
\hline 12. Espero amar... por toda minha vida. & 0,61 & $-0,02$ & 0,09 & 0,42 \\
\hline 19. Eu recebo considerável suporte emocional de... & $-0,04$ & 0,77 & 0,09 & 0,75 \\
\hline 38. Sinto que... realmente me compreende. & 0,03 & 0,69 & 0,16 & 0,62 \\
\hline 40. Sinto que realmente posso confiar em ... & 0,15 & 0,69 & $-0,15$ & 0,47 \\
\hline 6. Posso contar com... na hora que eu necessitar. & 0,05 & 0,69 & $-0,15$ & 0,46 \\
\hline 24. Me comunico bem com... & $-0,10$ & 0,57 & 0,28 & 0,62 \\
\hline 3. Só em olhar para... é excitante. & $-0,05$ & 0,03 & 0,70 & 0,73 \\
\hline 11. Acho... muito atraente. & 0,18 & 0,01 & 0,63 & 0,74 \\
\hline 23. Eu particularmente gosto do contato físico com... & 0,04 & 0,06 & 0,63 & 0,66 \\
\hline 37. Meu relacionamento com... é apaixonante. & 0,29 & 0,19 & 0,49 & 0,64 \\
\hline 5. Com frequência, durante o dia, me descubro pensando em... & 0,12 & 0,09 & 0,48 & 0,54 \\
\hline Variância comum explicada & 3,5 & 2,7 & 2,3 & \\
\hline Variância comum explicada (\%) & 41 & 32 & 27 & \\
\hline alfa de Cronbach & 0,91 & 0,84 & 0,80 & \\
\hline alfa ETAS-R & 0,92 & & & \\
\hline
\end{tabular}

Nota. G1=Amor, F1=Decisão/Compromisso, F2=Intimidade e F3=Paixão

Após a rotação os 15 itens carregaram mais forte nos fatores para os quais foram originalmente designados, e as cargas fatoriais saturadas nos outros fatores foram $<0,30$ (Tabela 3). Os alfas de Cronbach encontrados para a ETAS-R total e suas subescalas variaram de 0,92 a 0,80 , revelando elevada consistência interna para todas as dimensões do instrumento (Tabela 3). A correlação média interitens foi 0,42 , variando de 0,14 a 0,77 . A correlação item total variou de 0,45 a 0,77 . As correlações entre as três subescalas Paixão, Intimidade e Decisão/ Compromisso variaram de 0,58 a 0,67 , ou seja, moderadas fortes. As correlações entre as três subescalas e a escala total variaram de 0,84 a 0,87 , com níveis de significância estatística $<0,001$.

Tabela 4

Matriz de Cargas Fatoriais da Solução Schmid-Leiman para os Itens da ETAS-R

\begin{tabular}{ccccc}
\hline Itens & F1 (Decisão/Compromisso) & F2 (Intimidade) & F3 (Paixão) & G1 (Amor) \\
\hline 13 & $\mathbf{0 , 4 5}$ & $-0,02$ & $-0,01$ & $\mathbf{0 , 7 4}$ \\
33 & $\mathbf{0 , 4 2}$ & 0,06 & $-0,06$ & $\mathbf{0 , 7 4}$ \\
28 & $\mathbf{0 , 3 8}$ & 0,04 & $-0,00$ & $\mathbf{0 , 6 9}$ \\
32 & $\mathbf{0 , 3 8}$ & $-0,02$ & 0,18 & $\mathbf{0 , 7 5}$ \\
12 & $\mathbf{0 , 3 1}$ & $-0,02$ & 0,07 & $\mathbf{0 , 5 6}$ \\
19 & $-0,02$ & $\mathbf{0 , 5 1}$ & 0,07 & $\mathbf{0 , 6 0}$ \\
38 & 0,02 & $\mathbf{0 , 4 6}$ & $\mathbf{0 , 1 3}$ & $\mathbf{0 , 5 4}$ \\
40 & 0,08 & $\mathbf{0 , 5 5}$ & $\mathbf{0 , 4 7}$ \\
6 & 0,03 & $\mathbf{0 , 4 6}$ & $\mathbf{0 , 5 1}$ \\
24 & $-0,05$ & $\mathbf{0 , 4 6}, 12$ & $\mathbf{0 , 4 0}$ \\
5 & $-0,02$ & 0,38 & 0,22 & $\mathbf{0 , 4 6}$ \\
11 & 0,06 & 0,02 & $\mathbf{0 , 5 6}$ & $\mathbf{0 , 3 8}$ \\
37 & 0,09 & 0,06 & $\mathbf{0 , 5 4}$ & $\mathbf{0 , 4 6}$ \\
\hline G1 (Amor) & 0,02 & 0,01 & $\mathbf{0 , 5 8}$ & $\mathbf{0 , 3 9}$ \\
\hline
\end{tabular}


Os resultados da SSL revelaram que as cargas fatoriais do fator G1 de segunda ordem são mais elevadas do que as cargas fatoriais dos fatores F1, F2 e F3 de primeira ordem na maioria dos itens da ETAS-R. Excetuando apenas o item 3, que houve predomínio da carga fatorial do F3 sobre o G1. No entanto, as cargas fatoriais de todos os itens nas dimensões de primeira ordem saturaram de acordo com a designação original (Tabela 4).

\section{Discussão}

No estudo 1, foi observado que o primeiro fator não rotado extraído da ETAS teve amplíssimo predomínio sobre variância total dos escores. As três subescalas apresentaram forte dependência entre si e diversos itens revelaram superposições de cargas fatoriais em dois ou mais fatores. Como visto antes, esses resultados são frequentes nos relatos feitos por pesquisadores estrangeiros (Acker \& Davis, 1992; Aron \& Westbay, 1996; Carreño \& Serrano, 1995; Chojnacki \& Walsh, 1990; Hendrick \& Hendrick, 1989; Sternberg, 1997; Whitley, 1993) e nacionais (Cassep-Borges \& Teodoro, 2007; Cassepp-Borges \& Pasquali, 2012; Hernandez, 1999).

Hendrick e Hendrick (1989) e Carreño e Serrano (1995) sugeriram que a ETAS seja unidimensional e que a medida, no lugar de três componentes independentes, teria três facetas, tais como, a Escala de Amor Apaixonado de Hatfield e Sprecher (1986) que possui as facetas cognitivas, afetivas e comportamentais. No caso da ETAS, as facetas seriam de naturezas motivacionais (Paixão), afetivas (Intimidade) e cognitivas (Decisão/Compromisso), como identificou Sternberg (1984).

Os estudos realizados trouxeram a suposição de que a ETAS tenha uma dimensão geral. Isso está baseado, principalmente, nas elevadas correlações encontradas entre suas subescalas. Além disso, embora apresentem a consistência interna para a medida global, não trouxeram mais evidências da existência do fator geral (Cassepp-Borges \& Pasquali, 2012; Cassep-Borges \& Teodoro, 2007; Hernandez, 1999).

Gouveia et al. (2009) verificaram a possibilidade de um fator geral de amor para a ETAS-R mediante a realização de uma análise de componentes principais com o critério de extração de uma única dimensão. Esta apresentou autovalor de 7,61, explicando 50,7\% da variância total, com saturações nos itens $>0,60$ e alfa de Cronbach de 0,93 . Os pesquisadores sugeriram a possibilidade de ser considerada essa pontuação total, embora a teoria suponha três fatores de primeira ordem.

Andrade et al. (2013) testaram um modelo teórico para os escores da ETAS-R de Cassepp-Borges e
Teodoro (2007) mediante análise fatorial confirmatória (AFC) de um modelo que não contemplou a dimensão geral. Cassepp-Borges e Pasquali (2014) também testaram, por meio de AFC, dois modelos para a mesma versão da ETAS-R: um com as subescalas intercorrelacionadas e outro com um fator geral explicando os três fatores. Como os índices de ajuste estimados foram os mesmos para ambos os modelos testados, os pesquisadores optaram pelo segundo que atende à teoria de Sternberg (1986).

A análise fatorial hierárquica é um procedimento muito pouco utilizado no desenvolvimento e avaliação de instrumentos psicológicos. Soluções hierárquicas provavelmente são apropriadas para muitos instrumentos psicológicos de construtos que são compostos de múltiplas facetas correlacionadas. Contudo, essa técnica estatística não tem sido muito utilizada pelos pesquisadores (Laros, 2005).

No presente estudo, os resultados da SSL indicaram que a ETAS possui um forte fator de segunda ordem. Dos 45 itens da ETAS, 43 apresentaram cargas mais fortes no fator geral do que nos três fatores específicos. Esses itens, que além de representar essa dimensão geral deveriam também representar a especificidade dos construtos que a compõem, não o fizeram com desempenho suficiente. A partir desses dados, conclui-se que, no uso da versão completa do instrumento, deveria ser considerada a medida global. Por outro lado, sugere-se que novas investidas possam revisar, alterar ou criar novos itens para que as representações das dimensões específicas possam realmente efetivar-se.

De outra forma, conforme os resultados do estudo 2, a ETAS-R mostrou-se uma medida competente na representação das dimensões geral e específica. Por outro lado, embora as reduções de itens possam promover a superação de problemas psicométricos, certamente geram novos, tais como as perdas de aspectos importantes dos conteúdos dos construtos. Conforme Pasquali (2003), o número de itens de um instrumento estará relacionado à necessidade de representar operacional $\mathrm{e}$ extensivamente a totalidade ou a maior parte da amplitude do construto. Portanto, a redução do número de itens numa medida trará perdas de conteúdo dos conceitos que estão sendo representados por eles. Versões reduzidas poderão viabilizar o trabalho em algumas situações complexas da pesquisa, como de múltiplas amostras ou medidas.

As amostras atuais não probabilísticas e restritas a duas regiões do país, não podem ter a pretensão de representar qualquer população, portanto, os resultados encontrados devem ser compreendidos nessa perspectiva. Novas investidas com escores de sujeitos de outras regiões brasileiras poderão avançar na tarefa de gerar mais evidências e contribuir para a clarificação da estrutura fatorial da ETAS. 


\section{Referências}

Acker, M., \& Davis, M. H. (1992). Intimacy, passion and commitment in adult romantic relationships: A test of the triangular theory of love. Journal of Social and Personal Relationships, 9(1), 21-50. doi: 10.1177/0265407592091002

Andrade, A. L., Garcia, A., \& Cassep-Borges, V. (2013). Evidências de validade da Escala Triangular do Amor de Sternberg - Reduzida (ETAS-R). Psico-USF, 18(3), 501-510. doi:10.1590/S1413-82712013000300016

Aron, A., \& Westbay, L. (1996). Dimensions of the prototype of love. Journal of Personality and Social Psychology, 70(3), 535-551. doi:10.1037/0022-3514.70.3.535

Carreño, M., \& Serrano, G. (1995). Analisis de instrumentos para la medida del amor. Revista de Psicologia Social, 10(2), $131-148$.

Cassepp-Borges, V., \& Pasquali, L. (2012). Estudo nacional dos atributos psicométricos da escala triangular do amor de Sternberg. Paidéia, 22(51), 21-31. doi:10.1590/S0103-863X2012000100004

Cassepp-Borges, V., \& Pasquali, L. (2014). A redução de itens como uma alternativa para a escala triangular do amor. Psicologia, 28(2), 11-20.

Cassepp-Borges, V., \& Teodoro, M. (2007). Propriedades psicométricas da versão brasileira da escala triangular do amor de Sternberg. Psicologia: Reflexão e Crítica, 20(3), 513-522. doi:10.1590/S010279722007000300020

Chojnacki, J. T., \& Walsh, W. B. (1990). Reliability and concurrent validity of the Sternberg triangular love scale. Psychological Reports, 67(1), 219-224. doi: 10.1177/0265407593103013

Gouveia, V. V., Fonseca, P. N. da, Cavalcanti, J. P. N., Diniz, P. K. da C., \& Dória, L. C. (2009). Versão abreviada da escala triangular do amor: evidências de validade fatorial e consistência interna. Estudos de Psicologia, 14(1), 31-39. doi:10.1590/S1413-294X2009000100005

Graham, J. M. (2011). Measuring love in romantic relationships: A meta-analysis. Journal of Social and Personal Relationships, 28(6), 748-771. doi: 10.1177/0265407510389126

Hair, J. F., Black, W. C., Babin, B. J., Anderson, R. E., \& Tatham, R. L. (2009). Análise multivariada de dados. Porto Alegre: Bookman.

Hatfield, E., \& Sprecher, S. (1986). Measuring passionate love in intimate relations. Journal of Adolescence, 9, 383-410.

Hendrick, C., \& Hendrick, S. S. (1986). A theory and method of love. Journal of Personality and Social Psychology, 50(2), $392-402$. doi: $10.1037 / 0022-3514.50 .2 .392$

Hendrick, C., \& Hendrick, S. S. (1989). Research on love: Does it measure up? Journal of Personality and Social Psychology, 56(5), 784-794. doi: 10.1037/0022-3514.56.5.784

Hernandez, J. A. E. (1999). Validação da estrutura da escala triangular do amor: análise fatorial confirmatória. Aletheia, 9, 15-25.

Horn, J. L. (1965). A rationale and test for the number of factors in factor analysis. Psychometrika, 30(2), 179-85. doi: 10.1007/BF02289447

Laros, J. A. (2005). O uso da análise fatorial: algumas diretrizes para pesquisadores. Em L. Pasquali (Ed.). Análise fatorial para pesquisadores (pp.163-184). Brasília: LabPAM.

Lemieux, R., \& Hale, J. L. (2000). Intimacy, passion, and commitment among married inviduals: Further testing of the triangular theory of love. Psychological Reports, 87, 941-948. doi: 10.2466/pr0.2000.87.3.941

Levinger, G., Rands, M., \& Talaber, R. (1977). The assessment of involvement and rewardingness in close and casual pair relationships, National Science Foundation Report DK. Amherst: University of Massachusetts Press.

Lorenzo-Seva, U., \& Ferrando, P. J. (2013). Factor 9.2: A comprehensive program for fitting exploratory and semiconfirmatory factor analysis and IRT models. Applied Psychological Measurement, 37(6), 497-498. doi: 10.1177/0146621613487794

Pasquali, L. (2003). Princípios de elaboração de escalas psicológicas. Revista de Psiquiatria Clínica, 25(5), 206-213.

Rubin, Z. (1970). Measurement of romantic love. Journal of Personality and Social Psychology, 16(2), 265-273. doi:10.1037/h0029841

Schmid, J., \& Leiman, J. N. (1957). The development of hierarchical factor solutions. Psychometrika, 22(1), 53-61. doi: 10.1007/BF02289209

Sternberg, R. J. (1986). A triangular theory of love. Psychological Review, 93(2), 119-135. doi: 10.1037/0033-295X.93.2.119

Sternberg, R. J. (1988). Triangulating love. Em R. J. Sternberg \& M. L. Barnes (Eds.), The psychology of love (pp. 119-138). New Haven, CT: Yale University Press.

Sternberg, R. (1989). El triángulo del amor: intimidade, amor, compromisso. Barcelona: Paidós.

Sternberg, R. J. (1997). A construct-validation of a triangular love scale. European Journal of Social Psychology, 27, 313-335. doi:10.1002/ (SICI)1099-0992(199705)27:3 <313:AID-EJSP824>3.0.CO;2-4

Sternberg, R. J. (1998). Cupid's arrow: The course of love through time. New York: Cambridge University Press.

Sternberg, R. J. (2006). A duplex theory of love. Em R. J. Sternberg \& K. Weis (Eds.), The New Psychology of Love (pp. 184-199). New Haven: Yale University Press.

Sternberg, R., \& Grajek, S. (1984). The nature of love. Journal of Personality and Social Psychology, 47(2), 312-329. doi: 10.1037/00223514.47.2.312

Whitley, B. E. (1993). Reliability and aspects of the construct validity of Sternberg's triangular love scale. Journal of Social and Personal Relationships, 10(3), 475-480. doi:10.1177/0265407593103013

Wolff, H-G., \& Preising, K. (2005). Exploring item and higher order factor structure with the Schmid-Leiman solution: Syntax codes for SPSS and SAS. Behavior Research Methods, 37(1), 48-58. doi: 10.3758/BF03206397

recebido em julho de 2015 reformulado em outubro de 2015 aprovado em novembro de 2015

\section{Sobre o autor}

José Augusto Evangelho Hernandez é Psicólogo e doutor em Psicologia do Desenvolvimento pela Universidade Federal do Rio Grande do Sul, com estágio pós-doutoral pela Universidade Federal do Rio Grande do Sul e pela Universidad Rovira i Virgili/ES. Professor do Programa de Pós-graduação em Psicologia Social da Universidade do Estado do Rio de Janeiro. 